\title{
Discrete Poincaré Lemma
}

\author{
Mathieu Desbrun \\ 256-80, Computer Science, Caltech, Pasadena, CA 91125. \\ Melvin Leok \\ Mathematics, University of Michigan, 525 East University, Ann Arbor, MI 48109. \\ Jerrold E. Marsden \\ 107-81, Control and Dynamical Systems, Caltech, Pasadena, CA 91125.
}

\begin{abstract}
This paper proves a discrete analogue of the Poincaré lemma in the context of a discrete exterior calculus based on simplicial cochains. The proof requires the construction of a generalized cone operator, $p: C_{k}(K) \rightarrow C_{k+1}(K)$, as the geometric cone of a simplex cannot, in general, be interpreted as a chain in the simplicial complex. The corresponding cocone operator $H: C^{k}(K) \rightarrow C^{k-1}(K)$ can be shown to be a homotopy operator, and this yields the discrete Poincaré lemma.

The generalized cone operator is a combinatorial operator that can be constructed for any simplicial complex that can be grown by a process of local augmentation. In particular, regular triangulations and tetrahedralizations of $\mathbb{R}^{2}$ and $\mathbb{R}^{3}$ are presented, for which the discrete Poincaré lemma is globally valid.
\end{abstract}

\section{Introduction}

The exactness properties of differential complexes such as the de Rham complex have recently been shown to play an important role in the design and stability of numerical methods for partial differential equations [3]. In computational electromagnetism, numerical schemes that discretize the de Rham com-

Email addresses: mathieu@cs.caltech.edu (Mathieu Desbrun), mleok@umich.edu (Melvin Leok), marsden@cds.caltech.edu (Jerrold E. Marsden).

Preprint submitted to Elsevier Science $\quad 16$ September 2004 
plex, using interpolation by Whitney forms, have become increasingly prevalent $[4,9]$, and yield generalizations of the Yee staggered-mesh algorithm [13].

The geometrical structure underlying these numerical schemes is that of exterior calculus, and in Desbrun, et.al. [5], we introduced a discrete exterior calculus based on simplicial cochains. The cochain representation is attractive as it is particularly simple, and avoids the need for interpolation of forms.

The cochain representation of discrete differential forms uses the formalism of simplicial cohomology (see Munkres [11]) and identifies a cochain with a discrete differential form, and the coboundary with the exterior derivative. Consequently, a discrete differential form can only be evaluated on a chain.

The standard proof of the Poincaré lemma involves the construction of a homotopy operator though the cocone construction. This construction is unsatisfactory for developing a combinatorial proof of the discrete Poincaré lemma, since there is no canonical way to express the combinatorial cone of a $k$-simplex as a chain consisting of existing $(k+1)$-simplices.

Only by choosing a geometric realization of the abstract simplicial complex does it make sense to ask whether the cone of a simplex is expressible as a chain in the original simplicial complex. Even if we chose a geometric realization of the abstract simplicial complex, we find that this does not, in general, yield a representation of the cone operator as a map from chains to chains.

As an example, consider the figure below. Given the simplicial complex on the left, consisting of triangles, edges and nodes, we wish, in the center figure, to consider the cone of the bold edge with respect to the top most node. Clearly, the resulting cone in the right figure, which is shaded grey, cannot be expressed as a combination of the triangles in the original complex. As such, we cannot express the geometric cone as a combinatorial map in an obvious way.
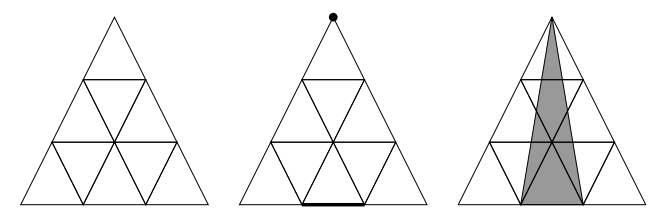

In this paper, a generalized cone operator that is valid for chains is developed with the essential homotopy properties to yield the discrete Poincaré lemma. In the rest of this section, we will review some basic results from simplicial algebraic topology, and relate them to discrete exterior calculus.

In the second section, we will first consider simplicial complexes on Euclidean space and introduce trivially star-shaped complexes, where the geometric cone operator is expressible as a map from $k$-chains to $(k+1)$-chains. Next, we will consider logically star-shaped complexes, which are simplicially isomorphic 
to trivially star-shaped complex. Here, the logical cone operator can be constructed by conjugating the standard cone operator with the isomorphism that relates the vertex scheme of the logically-star shaped simplicial complex to the vertex scheme of the trivially star-shaped simplicial complex.

Finally, drawing upon intuition developed in studying trivially star-shaped complexes, we will construct a generalized cone operator that is valid for any simplicial complex that can be grown by a process of local augmentation. The discrete Poincaré lemma is valid for these generalized star-shaped complexes, which include regular triangulations and tetrahedralizations of $\mathbb{R}^{2}$ and $\mathbb{R}^{3}$.

For discrete analogues of the Poincaré lemma which are obtained through the use of interpolated forms, the reader is referred to the work of Bossavit [4] and Hiptmair [8]. Discrete homotopy operators in the context of logically rectangular meshes were addressed in Hydon and Mansfield [10].

\subsection{Primal Simplicial Complex}

To discretize a continuous problem using discrete exterior calculus, we first discretize the manifold as a simplicial complex. This is typically a simplicial complex in Euclidean space, but it could also be an abstract simplicial complex. This is sufficient in this paper as differential forms are metric-independent, but the full theory of discrete exterior calculus [5] requires a local metric.

We will now recall some basic definitions of simplices, simplicial complexes and abstract simplicial complexes, which are standard from algebraic topology. A more extensive treatment can be found in Chapter $1, \S 1$ to $\S 3$ of Munkres [11].

Definition 1 A $k$-simplex is the convex span of $k+1$ geometrically independent points,

$$
\sigma^{k}=\left[v_{0}, v_{1}, \ldots, v_{k}\right]=\left\{\sum_{i=0}^{k} \alpha^{i} v_{i} \mid \alpha^{i} \geq 0, \sum_{i=0}^{n} \alpha^{i}=1\right\}
$$

The points $v_{0}, \ldots, v_{k}$ are the vertices of the simplex, and $k$ is the dimension of the simplex. Any simplex spanned by a (proper) subset of $\left\{v_{0}, \ldots, v_{k}\right\}$ is a (proper) face of $\sigma^{k}$. If $\sigma^{l}$ is a proper face of $\sigma^{k}$ then we write $\sigma^{l} \prec \sigma^{k}$.

Example 2 Consider 3 noncolinear points $v_{0}, v_{1}$ and $v_{2}$ in $\mathbb{R}^{3}$. Then these three points individually are examples of 0-simplices which are assumed to have no orientation. Examples of 1-simplices are the oriented line segments $\left[v_{0}, v_{1}\right],\left[v_{1}, v_{2}\right]$ and $\left[v_{0}, v_{2}\right]$. By writing the vertices in that order we have given orientations to these 1-simplices, i.e. $\left[v_{0}, v_{1}\right]$ is oriented from $v_{0}$ to $v_{1}$. The triangle $\left[v_{0}, v_{1}, v_{2}\right]$ is a 2-simplex oriented in counter clockwise direction. Note 
that the orientation of $\left[v_{0}, v_{2}\right]$ does not agree with that of the triangle.

Definition 3 A simplicial complex $K$ in $\mathbb{R}^{N}$ is a collection of simplices in $\mathbb{R}^{N}$ such that,

(1) Every face of a simplex of $K$ is in $K$.

(2) The intersection of any two simplices of $K$ is a face of each of them.

Definition 4 The polytope of $K$, denoted $|K|$, is the geometric union of the simplices of $K$. A simplicial triangulation of a polytope $|K|$ is a simplicial complex $K$ such that the union of the simplices of $K$ recover the polytope $|K|$.

Definition 5 If $L$ is a subcollection of $K$ that contains all faces of its elements, then $L$ is a simplicial complex in its own right, and it is called a subcomplex of $K$. The collection of all simplices of $K$ of dimension at most $k$, is a subcomplex which is called the $k$-skeleton of $K$ and is denoted $K^{(k)}$.

We now introduce the notion of an abstract simplicial complex, which captures the topology of a simplicial complex by encoding its connectivity.

Definition 6 An abstract simplicial complex is a collection $\mathcal{S}$ of finite nonempty sets, such that if $A$ is in $\mathcal{S}$, so is every nonempty subset of $A$.

An element $A$ of $\mathcal{S}$ is a simplex of $\mathcal{S}$, and its dimension is one less than the number of elements. Every nonempty subset of $A$ is a face of $A$. The dimension of $\mathcal{S}$ is the largest dimension of its simplices. The vertex set $V$ of $\mathcal{S}$ is the union of the one-point elements of $\mathcal{S}$, and the vertices $v \in V$ are identified with the 0 -simplices $\{v\} \in \mathcal{S}$. A subcollection of $\mathcal{S}$ that is itself a complex is called a subcomplex of $\mathcal{S}$.

Two abstract simplicial complexes $\mathcal{S}$ and $\mathcal{T}$ are isomorphic if there is a bijection $f$ mapping the vertex set of $\mathcal{S}$ to the vertex set of $\mathcal{T}$ such that $\left\{v_{0}, \ldots, v_{k}\right\} \in \mathcal{S}$ if and only if $\left\{f\left(v_{0}\right), \ldots, f\left(v_{k}\right)\right\} \in \mathcal{T}$.

Definition 7 If $K$ is a simplicial complex, and $V$ is its vertex set, let $\mathcal{K}$ be the collection of all subsets $\left\{v_{0}, \ldots, v_{k}\right\}$ of $V$ such that $\left[v_{0}, \ldots, v_{k}\right] \in K$. Then, $\mathcal{K}$ is an abstract simplicial complex called the vertex scheme of $K$.

Definition $8 K$ is a geometric realization of $\mathcal{S}$ if the abstract simplicial complex $\mathcal{S}$ is isomorphic to the vertex scheme of the simplicial complex $K$.

As with simplicial complexes, we will denote the orientation of a simplex in an abstract simplicial complex using the ordered representation $\left[v_{0}, \ldots v_{k}\right]$. 


\subsection{Differential Forms and Exterior Derivative}

We will now define discrete differential forms. We will use some terms (which we will define) from algebraic topology but it will become clear by looking at the examples that one can gain a clear and working notion of what a discrete form is without any algebraic topology. We start with a few definitions for which more details can be found on page 26 and 27 of Munkres [11].

Definition 9 Let $K$ be a simplicial complex. We denote the free abelian group generated by a basis consisting of oriented $k$-simplices by, $C_{k}(K ; \mathbb{Z})$. This $i s$ the space of finite formal sums of the $k$-simplices, with coefficients in $\mathbb{Z}$. Elements of $C_{k}(K ; \mathbb{Z})$ are called $k$-chains.

\section{Example 10}
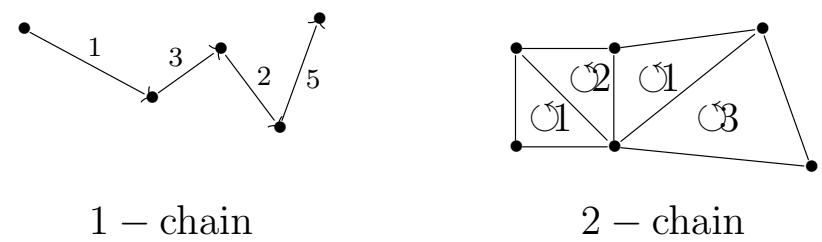

We view discrete $k$-forms as maps from the space of $k$-chains to $\mathbb{R}$. Recalling that the space of $k$-chains is a group we require the maps that define the forms to be homomorphisms into the additive group $\mathbb{R}$. Thus, discrete forms are cochains in algebraic topology. We will define cochains below in the definition of forms but for more context, and more details, readers can refer to any algebraic topology text, for example page 251 of Munkres [11].

This point of view of forms as cochains is not new. The idea of defining forms as cochains appears, for example, in the works of Adams [2], Dezin [6], Hiptmair [7], Sen et al.[12]. Our point of departure is that the other authors go on to develop a theory of discrete exterior calculus of forms by using interpolation which we will be able to avoid. The formal definition of discrete forms follows.

Definition 11 A primal discrete $k$-form $\alpha$ is a homomorphism from the chain group $C_{k}(K ; \mathbb{Z})$ to the additive group $\mathbb{R}$. Thus, a discrete $k$-form is an element of $\operatorname{Hom}\left(C_{k}(K), \mathbb{R}\right)$ the space of cochains. This space becomes an abelian group if we add two homomorphisms by adding their values in $\mathbb{R}$. The standard notation for $\operatorname{Hom}\left(C_{k}(K), \mathbb{R}\right)$ in algebraic topology is $C^{k}(K ; \mathbb{R})$. But we will often use the notation $\Omega_{d}^{k}(K)$ for this space as a reminder that this is the space of discrete (hence the $d$ subscript) $k$-forms on the simplicial complex K. Thus

$$
\Omega_{d}^{k}(K):=C^{k}(K ; \mathbb{R})=\operatorname{Hom}\left(C_{k}(K), \mathbb{R}\right)
$$


Note that by the above definition for $k$-chain $\sum_{i} a_{i} c_{i}^{k}$ (where $a_{i} \in \mathbb{Z}$ ) and a discrete $k$-form $\alpha$

$$
\alpha\left(\sum_{i} a_{i} c_{i}^{k}\right)=\sum_{i} a_{i} \alpha\left(c_{i}^{k}\right)
$$

and for two discrete $k$-forms $\alpha, \beta \in \Omega_{d}^{k}(K)$ and $k$-chain $c \in C_{k}(K ; \mathbb{Z})$

$$
(\alpha+\beta)(c)=\alpha(c)+\beta(c) .
$$

In exterior calculus on smooth manifolds, integration of $k$-forms on a $k$ manifold is defined in terms of integration in $\mathbb{R}^{k}$. This is done by doing the integration in local coordinates, which is independent of the choice of charts by the change of variables theorem. For details, see the first few pages of Chapter 7 of Abraham, Marsden and Ratiu [1]. We will not try to introduce the notion of integration of discrete forms on a simplicial complex. Instead, we will work with the natural bilinear pairing of cochains and chains defined by evaluation. More formally we have the following definition.

Definition 12 The natural pairing of a $k$-form $\alpha$ and $a k$-chain $c$ is defined as the bilinear pairing

$$
\langle\alpha, c\rangle=\alpha(c)
$$

As mentioned above, in discrete exterior calculus this natural pairing plays the role that integration of forms on chains plays in the usual exterior calculus. The two are related by a procedure done at the time of discretization. Indeed consider a simplicial triagulation $K$ of a polyhedron in $\mathbb{R}^{n}$ i.e consider a "flat" discrete manifold. Consider a continuous problem with some smooth forms defined in the space $|K| \subset \mathbb{R}^{n}$. To define the discrete form $\alpha_{d}^{k}$ corresponding to $\alpha^{k}$, one integrates $\alpha^{k}$ on all the $p$-simplices in $K$. Then the evaluation of $\alpha_{d}^{p}$ on a $k$-simplex $\sigma^{p}$ is defined by $\alpha_{d}^{k}\left(\sigma^{k}\right):=\int_{\sigma^{k}} \alpha^{k}$. Thus discretization is the only place where integration plays a role in our discrete exterior calculus.

Now we can define the discrete exterior derivative which we will call $\mathbf{d}$ as in the usual exterior calculus. The discrete exterior derivative will be defined as the dual with respect to the natural pairing defined above, of the boundary operator which is defined below.

Definition 13 The boundary operator $\partial_{k}: C_{k}(K ; \mathbb{Z}) \rightarrow C_{k-1}(K ; \mathbb{Z})$ is a homomorphism defined by defining it on a simplex $\sigma^{k}=\left[v_{0}, \ldots, v_{k}\right]$,

$$
\partial_{k} \sigma^{p}=\partial_{k}\left(\left[v_{0}, v_{1}, \ldots, v_{k}\right]\right)=\sum_{i=0}^{k}(-1)^{i}\left[v_{0}, \ldots, \hat{v}_{i}, \ldots, v_{k}\right]
$$

where $\left[v_{0}, \ldots, \hat{v}_{i}, \ldots, v_{k}\right]$ is the $(k-1)$-simplex obtained by omitting the vertex $v_{i}$. Note that $\partial_{k} \circ \partial_{k+1}=0$. 
Example 14 Given an oriented triangle $\left[v_{0}, v_{1}, v_{2}\right]$ the boundary, by the above, is $\left[v_{1}, v_{2}\right]-\left[v_{0}, v_{2}\right]+\left[v_{0}, v_{1}\right]$ which are the boundary edges of the triangle.

Definition 15 On a simplicial complex of dimension $n$, a chain complex is a collection of chain groups and homomorphisms $\partial_{k}$ such that

$0 \longrightarrow C_{n}(K) \stackrel{\partial_{n}}{\longrightarrow} \cdots \stackrel{\partial_{k+1}}{\longrightarrow} C_{k}(K) \stackrel{\partial_{k}}{\longrightarrow} \cdots \stackrel{\partial_{1}}{\longrightarrow} C_{0}(K) \quad \longrightarrow 0$,

and $\partial_{k} \circ \partial_{k+1}=0$.

Definition 16 The coboundary operator $\delta^{k}: C^{k}(K) \rightarrow C^{k+1}(K)$ defined by duality to the boundary operator using the natural bilinear pairing between discrete forms and chains. Specifically, for a discrete form $\alpha^{k} \in \Omega_{d}^{k}(K)$ and a chain $c_{k+1} \in C_{k+1}(K ; \mathbb{Z})$ we define $\delta^{k}$ by

$$
\left\langle\delta^{k} \alpha^{k}, c_{k+1}\right\rangle=\left\langle\alpha^{k}, \partial_{k+1} c_{k+1}\right\rangle
$$

that is

$$
\delta^{k}\left(\alpha^{k}\right)=\alpha^{k} \circ \partial_{k+1} .
$$

This definition of the coboundary operator induces the cochain complex,

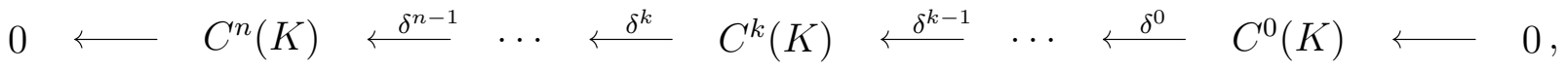
where it is easy to see that $\delta^{k+1} \circ \delta^{k}=0$.

Definition 17 The discrete exterior derivative denoted by $\mathbf{d}: \Omega_{d}^{k}(K) \rightarrow$ $\Omega_{d}^{k+1}(K)$ is defined to be the coboundary operator $\delta^{k}$.

Remark 18 With the above definition of the exterior derivative $\mathbf{d}: \Omega_{d}^{k}(K) \rightarrow$ $\Omega_{d}^{k+1}(K)$ and the relationship between the natural pairing and integration one can regard equation 1 as a discrete generalized Stokes' theorem. Thus given a $k$-chain $c$ and a discrete $k$-form $\alpha$ the discrete Stokes' theorem which is true by definition states that,

$$
\langle\mathbf{d} \alpha, c\rangle=\langle\alpha, \partial c\rangle
$$

Furthermore, it also follows immediately that, $\mathbf{d}^{2}=0$.

\section{Discrete Poincaré Lemma}

In this section, we will prove the discrete Poincaré lemma using the cocone construction. We will first consider the case of simplicial complexes that are trivially star-shaped, followed by logically star-shaped abstract complexes, 
before generalizing the result to generalized star-shaped abstract complexes.

As we have shown in the introduction, the cone is not a well-defined map from chains to chains for arbitrary simplicial complexes, and we will first consider trivially star-shaped complexes for which the cone is a well-defined map from chains to chains, before extending the construction to more general complexes.

Definition 19 Given a $k$-simplex $\sigma^{k}=\left[v_{0}, \ldots, v_{k}\right]$ we construct the cone with vertex $w$ and base $\sigma^{k}$, as follows,

$$
w \diamond \sigma^{k}=\left[w, v_{0}, \ldots, v_{k}\right]
$$

Lemma 20 The geometric cone operator satisfies the following property,

$$
\partial\left(w \diamond \sigma^{k}\right)+w \diamond\left(\partial \sigma^{k}\right)=\sigma^{k}
$$

PROOF. This is a standard result from simplicial algebraic topology.

\subsection{Trivially Star-Shaped Complexes}

We first introduce the notion of a trivially star-shaped complex, for which the standard cone construction yields a well-defined map from chains to chains, and for which the standard proof of the Poincaré lemma extends to the discrete case. We will also construct an arbitrarily dense tetrahedralization of an open neighborhood about a point using trivially star-shaped complexes.

It should be noted that the notion of a trivially star-shaped complex depends on the geometric realization, and is therefore not an intrinsic property of an abstract simplicial complex. Intrinsic generalizations will be considered in the next two subsections.

Definition 21 A complex $K$ is called trivially star-shaped if there exists a vertex $w \in K^{(0)}$ such that for all $\sigma^{k} \in K$, the cone with vertex $w$ and base $\sigma^{k}$ is (geometrically) expressible as a chain in $K$. That is to say,

$$
\exists w \in K^{(0)} \mid \forall \sigma^{k} \in K, w \diamond \sigma^{k} \in C_{k+1}(K) .
$$

We denote the cone operation with respect to $w$ as $p: C_{k}(K) \rightarrow C_{k+1}(K)$.

Lemma 22 In trivially star-shaped complexes, the cone operator $p: C_{k}(K) \rightarrow$ $C_{k+1}(K)$ satisfies the following identity,

$$
p \partial+\partial p=I
$$


at the level of chains.

PROOF. Follows from the identity for cones, and the fact that the cone is well-defined at the level of chains on trivially star-shaped complexes.

Definition 23 The cocone operator $H: C^{k}(K) \rightarrow C^{k-1}(K)$ is defined by,

$$
\left\langle H \alpha^{k}, \sigma^{k-1}\right\rangle=\left\langle\alpha^{k}, p\left(\sigma^{k-1}\right)\right\rangle .
$$

This operator is well-defined on trivially star-shaped simplicial complexes.

Lemma 24 The cocone operator $H: C^{k}(K) \rightarrow C^{k-1}(K)$ satisfies the following identity,

$$
H \mathbf{d}+\mathbf{d} H=I
$$

at the level of cochains.

PROOF. A simple duality argument applied to the cone identity,

$$
p \partial+\partial p=I
$$

yields the following,

$$
\begin{aligned}
\left\langle\alpha^{k}, \sigma^{k}\right\rangle & =\left\langle\alpha^{k},(p \partial+\partial p) \sigma^{k}\right\rangle \\
& =\left\langle\alpha^{k}, p \partial \sigma^{k}\right\rangle+\left\langle\alpha^{k}, \partial p \sigma^{k}\right\rangle \\
& =\left\langle H \alpha^{k}, \partial \sigma^{k}\right\rangle+\left\langle\mathbf{d} \alpha^{k}, p \sigma^{k}\right\rangle \\
& =\left\langle\left(\mathbf{d} H \alpha^{k}, \sigma^{k}\right\rangle+\left\langle H \mathbf{d} \alpha^{k}, \sigma^{k}\right\rangle\right. \\
& =\left\langle(\mathbf{d} H+H \mathbf{d}) \alpha^{k}, \sigma^{k}\right\rangle .
\end{aligned}
$$

Therefore,

$$
H \mathbf{d}+\mathbf{d} H=I
$$

at the level of cochains.

Corollary 25 (Discrete Poincaré Lemma for Trivially Star-shaped Complexes) Given a closed cochain $\alpha^{k}$, that is to say, $\mathbf{d} \alpha^{k}=0$, there exists a cochain $\beta^{k-1}$ such that $\mathbf{d} \beta^{k-1}=\alpha^{k}$.

PROOF. Applying the identity for cochains,

$$
H \mathbf{d}+\mathbf{d} H=I
$$

we have,

$$
\left\langle\alpha^{k}, \sigma^{k}\right\rangle=\left\langle(H \mathbf{d}+\mathbf{d} H) \alpha^{k}, \sigma^{k}\right\rangle
$$


but $\mathbf{d} \alpha^{k}=0$, so,

$$
=\left\langle\mathbf{d}\left(H \alpha^{k}\right), \sigma^{k}\right\rangle \text {. }
$$

Therefore, $\beta^{k-1}=H \alpha^{k}$ is such that $\mathbf{d} \beta^{k-1}=\alpha^{k}$ at the level of cochains.

Example 26 We construct an arbitrarily dense tetrahedralization of the cone of a $(n-1)$-simplex over the origin.

If we denote by $v_{i}^{k}$, the projection of the $v_{i}$ vertex to the $k$-th concentric sphere, where the 0-th concentric sphere is simply the central point, then we fill up the cone $\left[c, v_{1}, \ldots v_{n}\right]$ with simplices as follows,

$$
\left[v_{1}^{0}, v_{1}^{1}, \ldots, v_{n}^{1}\right],\left[v_{1}^{2}, v_{1}^{1}, \ldots, v_{n}^{1}\right],\left[v_{1}^{2}, v_{2}^{2}, v_{2}^{1}, \ldots, v_{n}^{1}\right], \ldots,\left[v_{1}^{2}, \ldots, v_{n}^{2}, v_{n}^{1}\right]
$$

Since $S^{n-1}$ is orientable, we use a consistent triangulation of $S^{n-1}$ and the $n$-cones to triangulate $B^{n}$ so that the resulting triangulation is star-shaped.

This fills up the region to the 1st concentric sphere, and we repeat the process by leapfrogging at the last vertex to add $\left[v_{1}^{2}, \ldots, v_{n}^{2}, v_{n}^{3}\right]$, and continuing the construction, to fill up the annulus between the 1st and 2nd concentric sphere. Thus, we can keep adding concentric shells to create an arbitrarily dense triangulation of a $n$-ball about the origin.

In three dimensions, these simplices are given by,

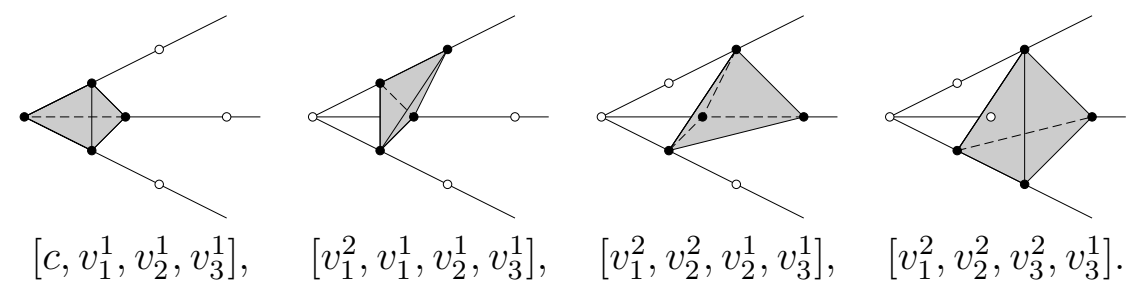

Putting them together, we obtain,

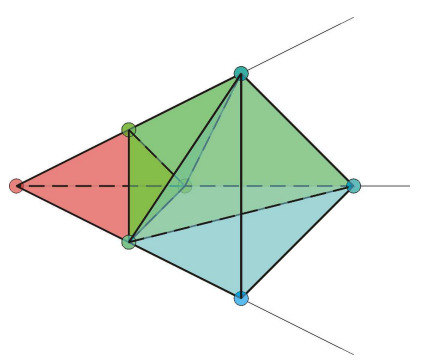

Triangulation of a 3-dimensional cone

This example is significant, since we can construct an arbitrarily dense trivially star-shaped triangulation of a $n$-ball, and recover the continuous Poincaré lemma from the discrete Poincaré lemma for trivially star-shaped complexes. 


\subsection{Logically Star-Shaped Complexes}

We now consider logically star-shaped complexes which are simplicially isomorphic to trivially star-shaped complexes, and as such inhert a cone operator with the desired homotopy properties from the trivially star-shaped complex. This property is intrinsic as it only depends on the vertex scheme of a complex.

Definition 27 A simplicial complex is logically star-shaped if its vertex scheme is isomorphic to the vertex scheme of a trivially star-shaped complex.

Example 28 Two simplicial complexes whose vertex schemes are isomorphic.

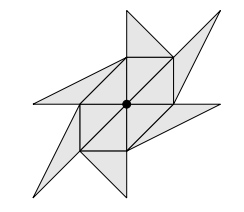

Trivially star-shaped complex

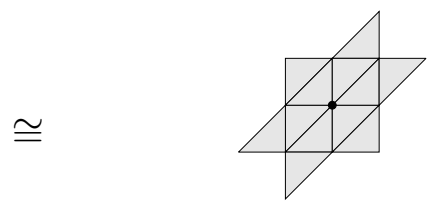

Logically star-shaped complex

Definition 29 The logical cone operator $p: C^{k}(L) \rightarrow C^{k+1}(L)$ satisfies,

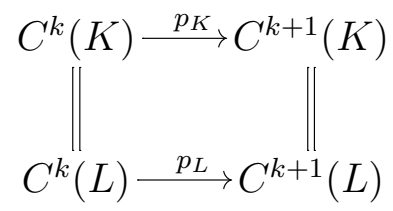

Which is to say that given the isomorphism $\varphi: K \rightarrow L$, we define,

$$
p_{L}=\varphi \circ p_{K} \circ \varphi^{-1}
$$

Example 30 An example of the construction of the logical cone operator.

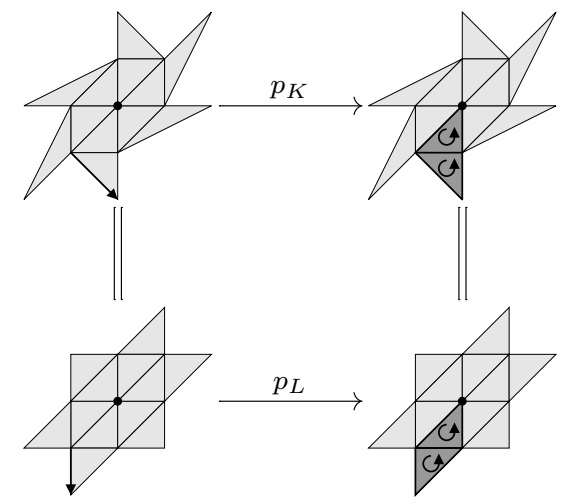

This definition of the logical cone operator results in the identities for the cone and cocone operator to follow from the trivially star-shaped case, and we record the results as follows. 
Lemma 31 In logically star-shaped complexes, the logical cone operator satisfies the following identity,

$$
p \partial+\partial p=I
$$

at the level of chains.

PROOF. Follows immediately by pushing forward the result for trivially star-shaped complexes using the isomorphism.

Lemma 32 In logically star-shaped complexes, the logical cocone operator satisfies the following identity,

$$
H \mathbf{d}+\mathbf{d} H=I
$$

at the level of cochains.

PROOF. Follows immediately by pushing forward the result for trivially star-shaped complexes using the isomorphism.

Thus, we have a discrete Poincaré lemma for logically star-shaped complexes.

Corollary 33 (Discrete Poincaré Lemma for Logically Star-shaped Complexes) Given a closed cochain $\alpha^{k}$, that is to say, $\mathbf{d} \alpha^{k}=0$, there exists a cochain $\beta^{k-1}$ such that $\mathbf{d} \beta^{k-1}=\alpha^{k}$.

PROOF. Follows from the above lemma using the proof for the trivially star-shaped case.

\subsection{Generalized Star-Shaped Complexes}

We now introduce generalized star-shaped complexes, which are constructed using a process of local augmentation. We can recursively construct a generalized cone operator such that it satisfies the homotopy identity,

$$
p \partial+\partial p=I
$$

which is the crucial property of the cone operator, from the point of view of proving the discrete Poincaré lemma.

Definition 34 Given a complex $K$, and a vertex $v$, the one-ring of $v$ is the set of all simplices of $K$ which contain $v$ as a vertex. 
Definition 35 Given a n-complex $K$, consider a vertex $w$ that is not already contained in the complex, and $a(n-1)$-chain $c_{n-1}$ that is contained on the boundary of $K$, and is included in the one-ring of some vertex on $\partial K$. Then, a one-ring cone augmentation of $K$ is the complex obtained by adding the $n$-cone $w \diamond c_{n-1}$, and all its faces to the complex.

Definition 36 A complex is generalized star-shaped if it can be constructed by repeated one-ring cone augmentation of an initially logically starshaped complex.

Note that the simplest example of a logically star-shaped complex is the complex consisting of an $n$-simplex and all its faces. We will now introduce an example of a regular triangulation of the plane that is generalized star-shaped.

Example 37 The regular 2-dimensional triangulation can be obtained by the successive application of the one-ring cone augmentation procedure, as the following sequence illustrates,

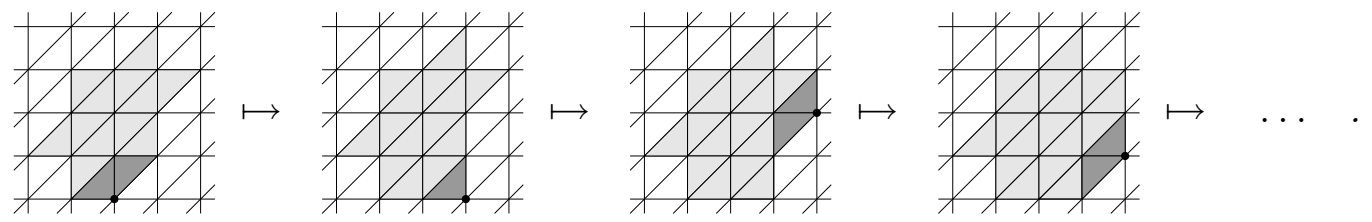

Remark 38 Since each simplex in the one-ring of $v$ contains $v$, and each simplex is connected, a chain consisting of simplices of a one-ring is connected. As such, a non-contractible complex cannot be constructed by inductive onering cone augmentation, as it will involve adding a cone $w \diamond c_{n-1}$, where $c_{n-1}$ is disjoint and therefore cannot be included in the one-ring of a vertex.

We construct our generalized cone operator $p: C^{k}(K) \rightarrow C^{k+1}(K)$ recursively. Since we are restricting ourselves to generalized star-shaped complexes, which are obtained by repeated one-ring augmentation of an initially logically starshaped complex, it suffices to show that we can define a generalized cone operator on the newly added simplices that satisfies the homotopy property.

We proceed by induction. The base case is a generalized star-shaped complex that is logically star-shaped, and has a logical cone operator which satisfies the homotopy property. The generalized cone operator is defined to coincide with the logical cone operator, and satisfies the homotopy property as well.

Given a generalized star-shaped complex $K_{i}$, we augment using one-ring cone augmentation by adding the $n$-cone $w \diamond c_{n-1}$, and all its faces to obtain $K_{i+1}$. We need to define $p$ for simplices in $K_{i+1} \backslash K_{i}$.

To define $p\left(\sigma^{k}\right)$ for $\sigma^{k} \in K_{i+1} \backslash K_{i}$, we choose a $\sigma^{k+1} \in K_{i+1} \backslash K_{i}$, to include in the cone $p\left(\sigma^{k}\right)$, such that $\sigma^{k+1} \succ \sigma^{k}$, and $\sigma^{k+1}$ and $\sigma^{k}$ are consistently 
oriented. Let us require that the homotopy property of $p$ holds on $\sigma^{k+1}$,

$$
\begin{aligned}
\sigma^{k+1} & =(p \partial+\partial p)\left(\sigma^{k+1}\right) \\
& =p\left(\partial \sigma^{k+1}\right)+\partial p\left(\sigma^{k+1}\right) \\
& =p\left(\partial \sigma^{k+1}-\sigma^{k}+\sigma^{k}\right)+\partial p\left(\sigma^{k+1}\right) \\
& =p\left(\sigma^{k}\right)+p\left(\partial \sigma^{k+1}-\sigma^{k}\right)+\partial p\left(\sigma^{k+1}\right) .
\end{aligned}
$$

The geometric cone operator suggests how to proceed. If a $(k+1)$-simplex $\sigma^{k}$ is part of the geometric cone of a $k$-simplex $\sigma^{k}$ with respect to the point $x$, then $\sigma^{k+1}$ is contained in a $(k+1)$-hyperplane containing $x$. Therefore, the geometric cone of $\sigma^{k+1}$ is $(k+1)$-dimensional. When the geometric cone is viewed as a map from $(k+1)$-chains to $(k+2)$-chains, it follows that $p\left(\sigma^{k+1}\right)=\varnothing$. Thus, if $\sigma^{k+1}$ is a term in $p\left(\sigma^{k}\right)$ for some $\sigma^{k}$, then $p\left(\sigma^{k+1}\right)=\varnothing$.

Since we chose to include $\sigma^{k+1}$ as one of the terms in $p\left(\sigma^{k}\right)$, let us follow the intuition suggested by the geometric cone and define $p\left(\sigma^{k+1}\right)=\varnothing$. Then,

$$
\begin{aligned}
\sigma^{k+1} & =p\left(\sigma^{k}\right)+p\left(\partial \sigma^{k+1}-\sigma^{k}\right)+\partial(\varnothing) \\
& =p\left(\sigma^{k}\right)+p\left(\partial \sigma^{k+1}-\sigma^{k}\right) .
\end{aligned}
$$

Rearranging, we obtain an expression for what $p\left(\sigma^{k}\right)$ needs to be,

$$
p\left(\sigma^{k}\right)=\sigma^{k+1}-p\left(\partial \sigma^{k+1}-\sigma^{k}\right) .
$$

To recap, we define $p\left(\sigma^{k}\right)$ by choosing $\sigma^{k+1} \succ \sigma^{k}$, such that $\sigma^{k+1}$ and $\sigma^{k}$ are consistently oriented. Then, we define,

$$
\begin{aligned}
p\left(\sigma^{k}\right) & =\sigma^{k+1}-p\left(\partial \sigma^{k+1}-\sigma^{k}\right), \\
p\left(\sigma^{k+1}\right) & =\varnothing .
\end{aligned}
$$

It remains to show that in defining $p\left(\sigma^{k}\right)$, we can order the definition of $p$ on the simplices of the cone so that the simplices in the chain $\partial \sigma^{k+1}-\sigma^{k}$ already have $p$ defined on it. If we can construct $p$ in the above fashion so that it is well-defined, the homotopy property will automatically hold by construction. We will now define the generalized cone operator for 2 and 3-dimensions.

Definition 39 In 2-dimensions, the 1-ring condition implies that the base of the cone consists of either one or two 1-simplices. To aid in visualization, consider the following diagram,

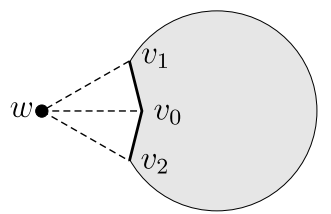

One-ring cone augmentation of a complex in 2-dimensions 
In the case of one 1-simplex, $\left[v_{0}, v_{1}\right]$, when we augment using the cone construction with the new vertex $w$, we define,

$$
\begin{aligned}
p([w]) & =\left[v_{0}, w\right]+p\left(\left[v_{0}\right]\right), & p\left(\left[v_{0}, w\right]\right) & =\varnothing, \\
p\left(\left[v_{1}, w\right]\right) & =\left[v_{0}, v_{1}, w\right]-p\left(\left[v_{0}, v_{1}\right]\right), & p\left(\left[v_{0}, v_{1}, w\right]\right) & =\varnothing .
\end{aligned}
$$

In the case of two 1-simplices, $\left[v_{0}, v_{1}\right],\left[v_{0}, v_{2}\right]$, we have,

$$
\begin{aligned}
p([w]) & =\left[v_{0}, w\right]+p\left(\left[v_{0}\right]\right), & p\left(\left[v_{0}, w\right]\right) & =\varnothing, \\
p\left(\left[v_{1}, w\right]\right) & =\left[v_{0}, v_{1}, w\right]-p\left(\left[v_{0}, v_{1}\right]\right), & & p\left(\left[v_{0}, v_{1}, w\right]\right)=\varnothing, \\
p\left(\left[v_{2}, w\right]\right) & =\left[v_{0}, v_{2}, w\right]-p\left(\left[v_{0}, v_{2}\right]\right), & & p\left(\left[v_{0}, v_{2}, w\right]\right)=\varnothing .
\end{aligned}
$$

Example 40 We will now compute the generalized cone operator for part of a regular 2-dimensional triangulation that is not logically star-shaped. Consider a logically star-shaped complex, and augment with a new vertex.

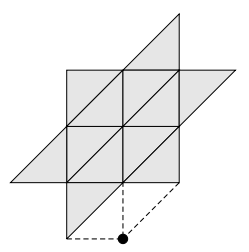

\section{Logically star-shaped complex augmented by cone}

We use the logical cone operator for the subcomplex that is logically starshaped, and the definition above for the newly introduced simplices. This yields,
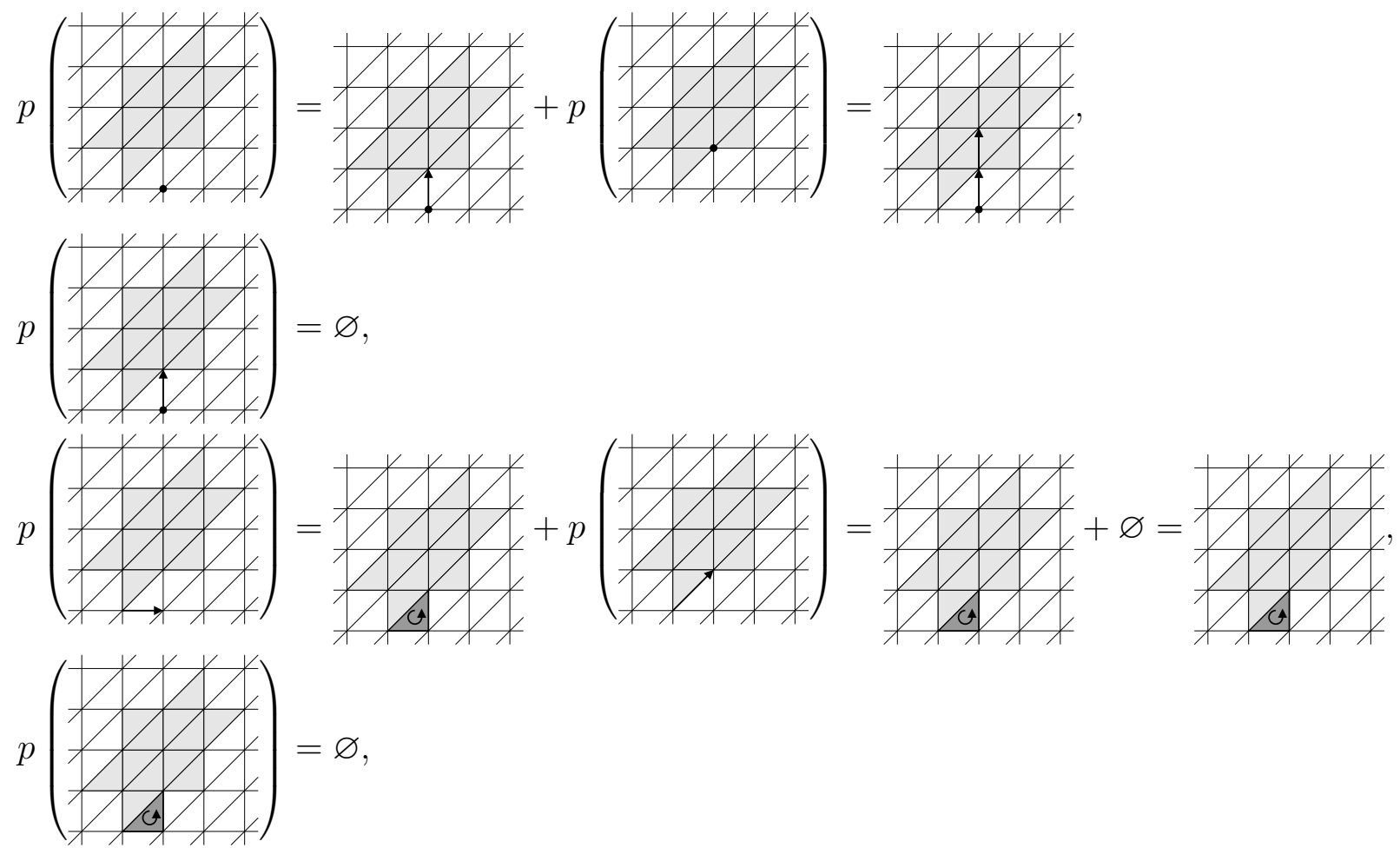

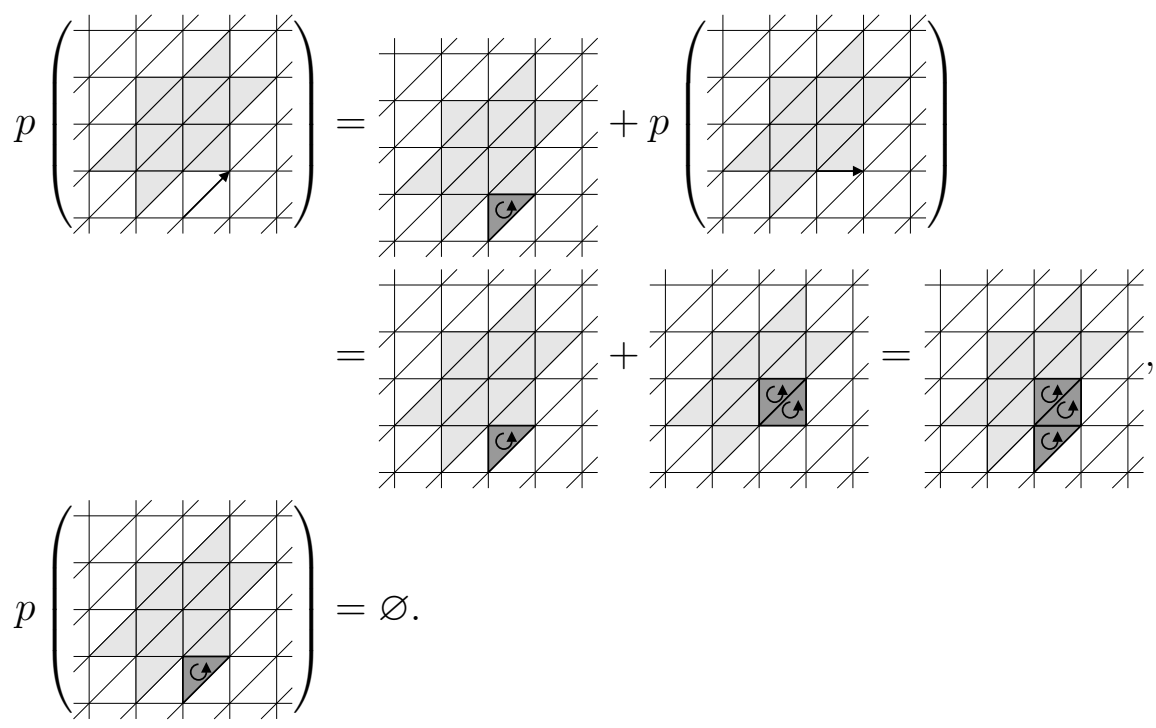

Definition 41 We now define the generalized cone operator in 3-dimensions. Denote by $v_{0}$ the center of the 1-ring on the 2-surface, to which we are augmenting the new vertex $w$. The other vertices of the 1-ring are enumerated in order $v_{1}, \ldots, v_{m}$. To aid in visualization, consider the following diagram,

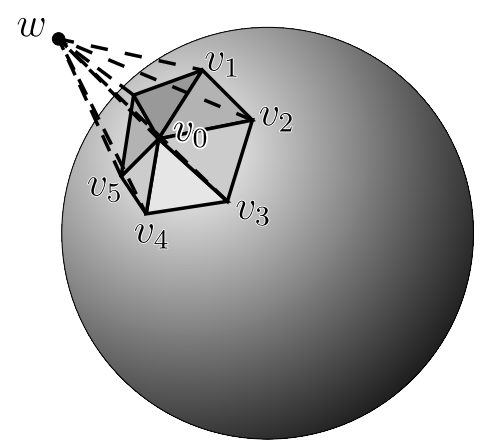

One-ring cone augmentation of a complex in 3-dimensions

If the 1-ring does not surround $v_{0}$, we denote the missing term by $\left[v_{0}, v_{1}, v_{m}\right]$.

$k=0$,

$$
p([w])=\left[v_{0}, w\right]+p\left(\left[v_{0}\right]\right), \quad p\left(\left[v_{0}, w\right]\right)=\varnothing
$$

$k=1$,

$$
\begin{aligned}
p\left(\left[v_{1}, w\right]\right) & =\left[v_{0}, v_{1}, w\right]-p\left(\left[v_{0}, v_{1}\right]\right), & p\left(\left[v_{0}, v_{1}, w\right]\right) & =\varnothing \\
p\left(\left[v_{m}, w\right]\right) & =\left[v_{0}, v_{m}, w\right]-p\left(\left[v_{0}, v_{m}\right]\right), & p\left(\left[v_{0}, v_{m}, w\right]\right) & =\varnothing
\end{aligned}
$$

$k=2$,

$$
p\left(\left[v_{1}, v_{2}, w\right]\right)=\left[v_{0}, v_{1}, v_{2}, w\right]+p\left(\left[v_{0}, v_{1}, v_{2}\right]\right), \quad p\left(\left[v_{0}, v_{1}, v_{2}, w\right]\right)=\varnothing
$$


$p\left(\left[v_{m-1}, v_{m}, w\right]\right)=\left[v_{0}, v_{m-1}, v_{m}, w\right]+p\left(\left[v_{0}, v_{m-1}, v_{m}\right]\right), \quad p\left(\left[v_{0}, v_{m-1}, v_{m}, w\right]\right)=\varnothing$

If it does go around completely,

$$
p\left(\left[v_{m}, v_{1}, w\right]\right)=\left[v_{0}, v_{m}, v_{1}, w\right]+p\left(\left[v_{0}, v_{m}, v_{1}\right]\right), \quad p\left(\left[v_{0}, v_{m}, v_{1}, w\right]\right)=\varnothing .
$$

Example 42 We provide a tetrahedralization of the unit cube that can be tiled to yield a regular tetrahedralization of $\mathbb{R}^{3}$. The 3-simplices are as follows,

$$
\begin{aligned}
& {\left[v_{000}, v_{001}, v_{010}, v_{10}\right],\left[v_{001}, v_{010}, v_{100}, v_{101}\right],\left[v_{001}, v_{010}, v_{011}, v_{101}\right],} \\
& {\left[v_{010}, v_{100}, v_{101}, v_{110}\right],\left[v_{010}, v_{011}, v_{101}, v_{110}\right],\left[v_{011}, v_{101}, v_{110}, v_{111}\right] .}
\end{aligned}
$$

The tetrahedralization of the unit cube can be visualized as follows,

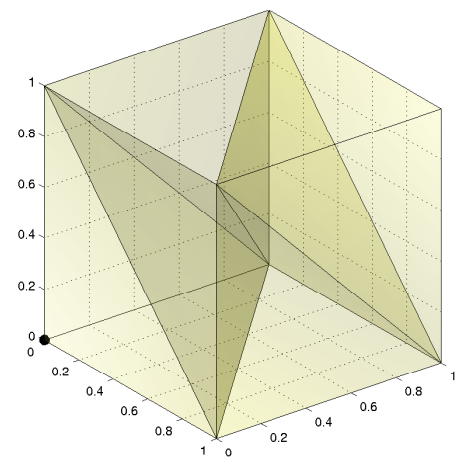

Tileable tetrahedralization of the unit cube

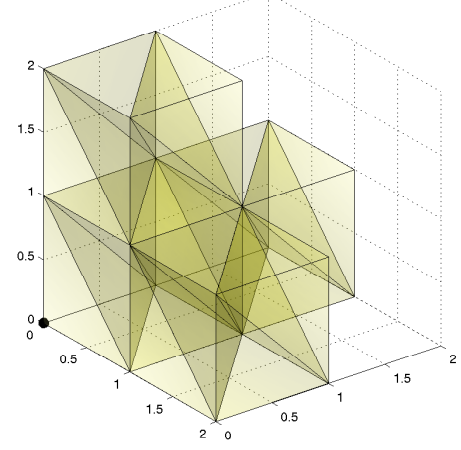

Partial tiling

Since this regular tetrahedralization can be constructed by the successive application of the one-ring cone augmentation procedure, the discrete Poincaré lemma can be extended to the entire regular tetrahedralization of $\mathbb{R}^{3}$.

In higher dimensions, we extend the construction of the generalized cone operator by choosing an appropriate enumeration of the base chain. The base chain is topologically the cone of $S^{n-2}$ (with possibly an open $n-2$ ball removed) with respect to the central point.

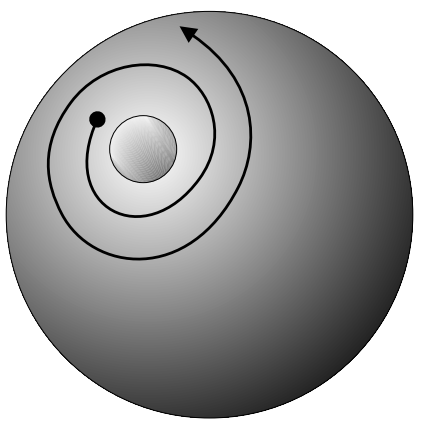

Spiral enumeration of $S^{n-2}, n=4$ 
By spiraling around $S^{n-2}$, starting from the boundary of the $n-2$ ball, and covering the rest of $S^{n-2}$, we obtain higher dimensional generalizations of Definitions 39, 41. Since $S^{2-2}=S^{0}$ is disjoint, $n=2$ is distinguished, and we were unable to use spiral enumeration of the simplices in 2-dimensions.

The generalized cone operator is constructed so that the homotopy property holds automatically.

Lemma 43 In generalized star-shaped complexes, the generalized cone operator satisfies the following identity,

$$
p \partial+\partial p=I
$$

at the level of chains.

PROOF. By construction of the generalized cone operator.

Lemma 44 In generalized star-shaped complexes, the generalized cocone operator satisfies the following identity,

$$
H \mathbf{d}+\mathbf{d} H=I
$$

at the level of cochains.

PROOF. Follows immediately from applying the proof in the trivially starshaped case, and using the identity in the previous lemma.

We have a discrete Poincaré lemma for generalized star-shaped complexes.

Corollary 45 (Discrete Poincaré Lemma for Generalized Star-shaped Complexes) Given a closed cochain $\alpha^{k}$, that is to say, $\mathbf{d} \alpha^{k}=0$, there exists a cochain $\beta^{k-1}$ such that $\mathbf{d} \beta^{k-1}=\alpha^{k}$.

PROOF. Follows from the above lemma using the proof for the trivially star-shaped case.

Example 46 We will show how the Poincaré lemma fails when the complex is not contractible. We consider a trivially star-shaped complex, and augment by one vertex so as to make it non-contractible. 


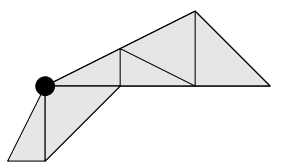

Trivially star-shaped complex

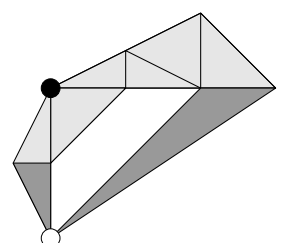

Non-contractible complex

When we attempt to verify the identity,

$$
p \partial+\partial p=I
$$

we see that it is only true up to a chain that is homotopic to the inner boundary.

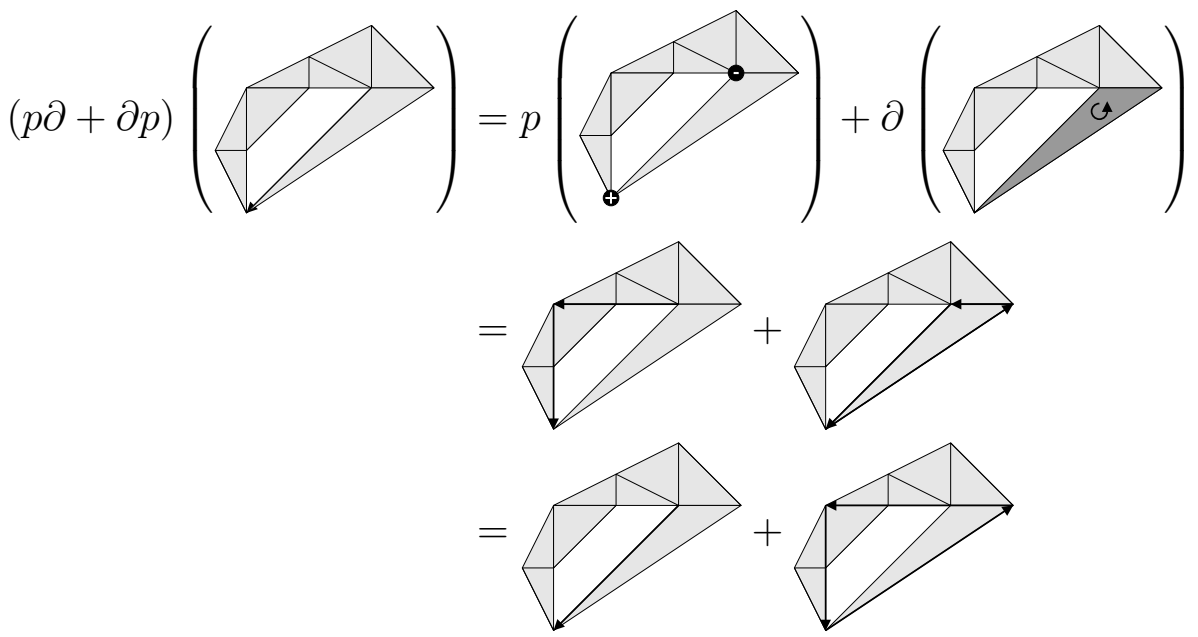

Since the second term is not the boundary of a 2-chain, it contributes a nontrivial term, even on closed discrete forms, and the Poincaré lemma breaks.

\section{Conclusion}

In summary, we have presented a constructive method of obtaining a local neighborhood in an unstructured mesh for which the discrete Poincaré lemma holds in the context of discrete exterior calculus. Furthermore, we introduced examples of regular space-filling triangulations and tetrahedralizations for which the exactness properties hold globally.

In the future, higher order analogues of the discrete theory of exterior calculus are desirable, but the cochain representation which assigns numerical quantities to a discrete set of geometric objects remains attractive due to its conceptual simplicity and the elegance of representing discrete operators as combinatorial operations on the mesh.

It is desirable to reconcile the two, by ensuring that higher-order interpolation 
and the combinatorial operations are consistent. This would yield more direct proofs of the exactness properties using the standard cocone construction on the interpolated differential form, while giving a discrete homotopy operator that could be efficiently realized as a combinatorial operation on the mesh.

\section{References}

[1] Abraham, R., Marsden, J.E. and Ratiu, T.S., Manifolds, Tensor Analysis and Applications, Springer Verlag, 1988.

[2] Adams, D.H., R-torsion and linking numbers from simplicial abelian gauge theories. e-print hep-th/9612009

[3] Arnold, D.N., Differential complexes and numerical stability, Proceedings of the International Congress of Mathematicians, Beijing 2002, Volume I: Plenary Lectures.

[4] Bossavit, A., Computational Electromagnetism: Variational Formulations, Complementarity, Edge Elements, Academic Press Series in Electromagnetism, Academic Press, 1998.

[5] Desbrun, M., A.N. Hirani, M. Leok, J.E. Marsden, Discrete Exterior Calculus, in preparation, 2003.

[6] Dezin, A.A., Multidimensional Analysis and Discrete Models, CRC Press, 1995.

[7] Hiptmair, R., Canonical Construction of Finite Elements, Math. Comp., 68, 1325-1346, 1999.

[8] Hiptmair, R., Higher Order Whitney Forms, Progress in Electromagnetics Research, PIER 32, 271-299, 2001.

[9] Hiptmair, R., Finite Elements in Computational Electromagnetism, Acta Numerica, 237-339, 2002.

[10] P.E. Hydon, Mansfield, E.L., A variational complex for difference equations, J. FoCM, Volume 4, Number 2, 187-217, 2004.

[11] Munkres, J.R., Elements of Algebraic Topology, Addison-Wesley, 1984.

[12] Sen, S., S. Sen, J.C. Sexton, D.H. Adams, Geometric discretization scheme applied to the Abelian Chern-Simons theory, Phys. Rev. E 61 3174-3185, 2000.

[13] K. Yee, Numerical solution of initial boundary value problems involving Maxwell's equations in isotropic media, IEEE Trans. Antennas and Propagation AP-14, 302-307, 1966. 